\title{
Evolving concepts on the management of dyslipidaemia
}

Olivier S. Descamps, Ann Verhaegen, Fabien Demeure, Michel Langlois, Ernst Rietzschel, Ann Mertens, Johan De Sutter, Caroline Wallemacq, Patrizio Lancellotti \& Guy De BackerOn behalf of the Belgian Society of Atherosclerosis, the Belgian Society of Cardiology, Young Cardiologists Club, the Royal Belgian Society of Laboratory Medicine, and the Belgian Working on Cardiovascular Prevention and Rehabilitation

To cite this article: Olivier S. Descamps, Ann Verhaegen, Fabien Demeure, Michel Langlois, Ernst Rietzschel, Ann Mertens, Johan De Sutter, Caroline Wallemacq, Patrizio Lancellotti \& Guy De BackerOn behalf of the Belgian Society of Atherosclerosis, the Belgian Society of Cardiology, Young Cardiologists Club, the Royal Belgian Society of Laboratory Medicine, and the Belgian Working on Cardiovascular Prevention and Rehabilitation (2019): Evolving concepts on the management of dyslipidaemia, Acta Clinica Belgica, DOI: 10.1080/17843286.2019.1702823

To link to this article: https://doi.org/10.1080/17843286.2019.1702823

Published online: 17 Dec 2019.

Submit your article to this journal $₫$

View related articles $\sqsubset$

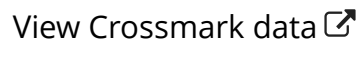




\title{
Evolving concepts on the management of dyslipidaemia
}

Olivier S. Descamps (10) ${ }^{\mathrm{a}}$, Ann Verhaegen ${ }^{\mathrm{b}}$, Fabien Demeurec, Michel Langlois ${ }^{\mathrm{d}}$, Ernst Rietzschele ${ }^{\mathrm{e}}$, Ann Mertens ${ }^{\mathrm{f}}$,

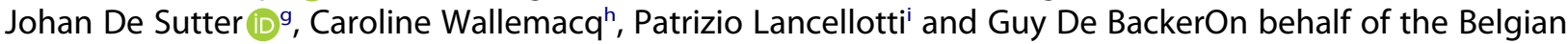
Society of Atherosclerosis, the Belgian Society of Cardiology, Young Cardiologists Club, the Royal Belgian Society of Laboratory Medicine, and the Belgian Working on Cardiovascular Prevention and Rehabilitation ${ }^{\mathrm{j}}$

\begin{abstract}
aDepartment of internal medicine, Centres Hospitaliers Jolimont, Haine Saint-Paul and department of cardiology, UCL Cliniques Universitaires Saint-

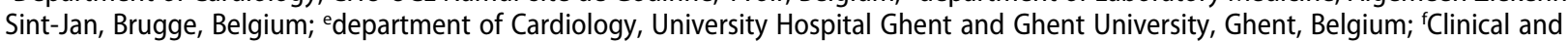
Experimental Endocrinology, Department of Chronic Diseases, Metabolism and Aging (CHROMETA), KU Leuven, Leuven, Belgium;

ABSTRACT

It has been well established that low-density lipoproteins (LDL) and other apolipoprotein B-containing lipoproteins are causally related to atherosclerotic cardiovascular disease (ASCVD) and that lowering these lipoproteins reduces the risk of ASCVD. By lowering LDL particles as much as possible, ASCVD can be prevented. There seems to be no LDL-cholesterol (LDL-C) threshold below which no further ASCVD prevention can be achieved. Furthermore, a low (an even very low) LDL-C appears to be safe. The new ESC/EAS guidelines based on these concepts are a step towards a benefit-based strategy by focusing on the clinical benefit that can be achieved by treating the cause of ASCVD. It is recommended to lower LDL-C as much as possible to prevent ASCVD, especially in high and very high-risk patients. With these new recommendations come recognition of the importance of combination therapies in high and very high-risk patients, first with statins and ezetimibe, and if needed with a PCSK9 inhibitor. The present paper is a review of some new concepts arising during the past 10 years in the field of lipidology and the description of what is new in the 2019 EAS/ESC guidelines.
\end{abstract}

Luc, Bruxelles, Belgium; bepartment of Endocrinology, Diabetology and Metabolism, Antwerp University Hospital, Antwerpen, Belgium; 'Department of Cardiology, CHU UCL Namur site de Godinne, Yvoir, Belgium; department of Laboratory Medicine, Algemeen Ziekenhuis פDepartment of Cardiology, AZ Maria Middelars Ghent and University Ghent, Belgium; ' ${ }^{2}$ department of Diabetes, Nutrition and Metabolic diseases, Centre Hospitalier Universitaire Sart Tilman, Liège, Belgium; 'GIGA Cardiovascular Sciences, department of Cardiology, Centre Hospitalier Universitaire Sart Tilman, Liège, Belgium; 'Gent Department of public health and primary care, Ghent University, Gent, Belgium

\section{KEYWORDS}

Cardiovascular prevention; cholesterol; coronary heart disease; genetic; atherosclerosis

\section{Major basic concepts developed during the past 10 years}

\section{LDL is a causal risk factor}

It has been well established that the association between LDL-C and ASCVD is not only statistically significant but that it is causal in origin (Figure 1) [1]. In medicine, the scientific evidence of a causal link between a factor and a disease is based on a number of criteria (called the 'Bradford-Hill criteria') including the strength of the association, the reversibility of risk through reduction of the factor by intervention, the time sequence where the factor precedes the disease, the reproducibility of the evidence (studies must be repeated many times and on large cohorts) and the consistency between different approaches. Regarding the strength of association, countless data from human epidemiological observations and their meta-analyses show the very strong link between high LDL-C levels and the risk of ASCVD [2].

Subsequently, in numerous randomized controlled trials and in their meta-analyses [3] it has been shown that the reduction of LDL-C with statins or with statins in combination with other LDL-C-lowering drugs leads to a reduction in the incidence of ASCVD. Data from genetic models of hypercholesterolemia such as familial hypercholesterolemia $(\mathrm{FH})$ show the dangerous nature of carrying very high levels of LDL-C from birth onwards.

Finally, the study of genetic variants, more frequent but less severe than FH (polymorphisms in genes involved in the production, absorption and elimination of cholesterol), in Mendelian randomization studies shows that small variations in LDL-C (e.g. $11 \mathrm{mg} / \mathrm{dL}$ for one variant of NPC1L1, the gene of the protein participating to the intestinal absorption of cholesterol) from birth onwards are sufficient to significantly reduce the risk of cardiovascular (CV) events throughout life (in this example, by 20\%). Mechanistically, the pathogenesis of atherosclerosis is very well understood as illustrated in Figure 1.

Thus, all these observations (strong association, CV benefit of therapeutic interventions that reduce LDL-C and time sequence confirmed by genetics) converge 


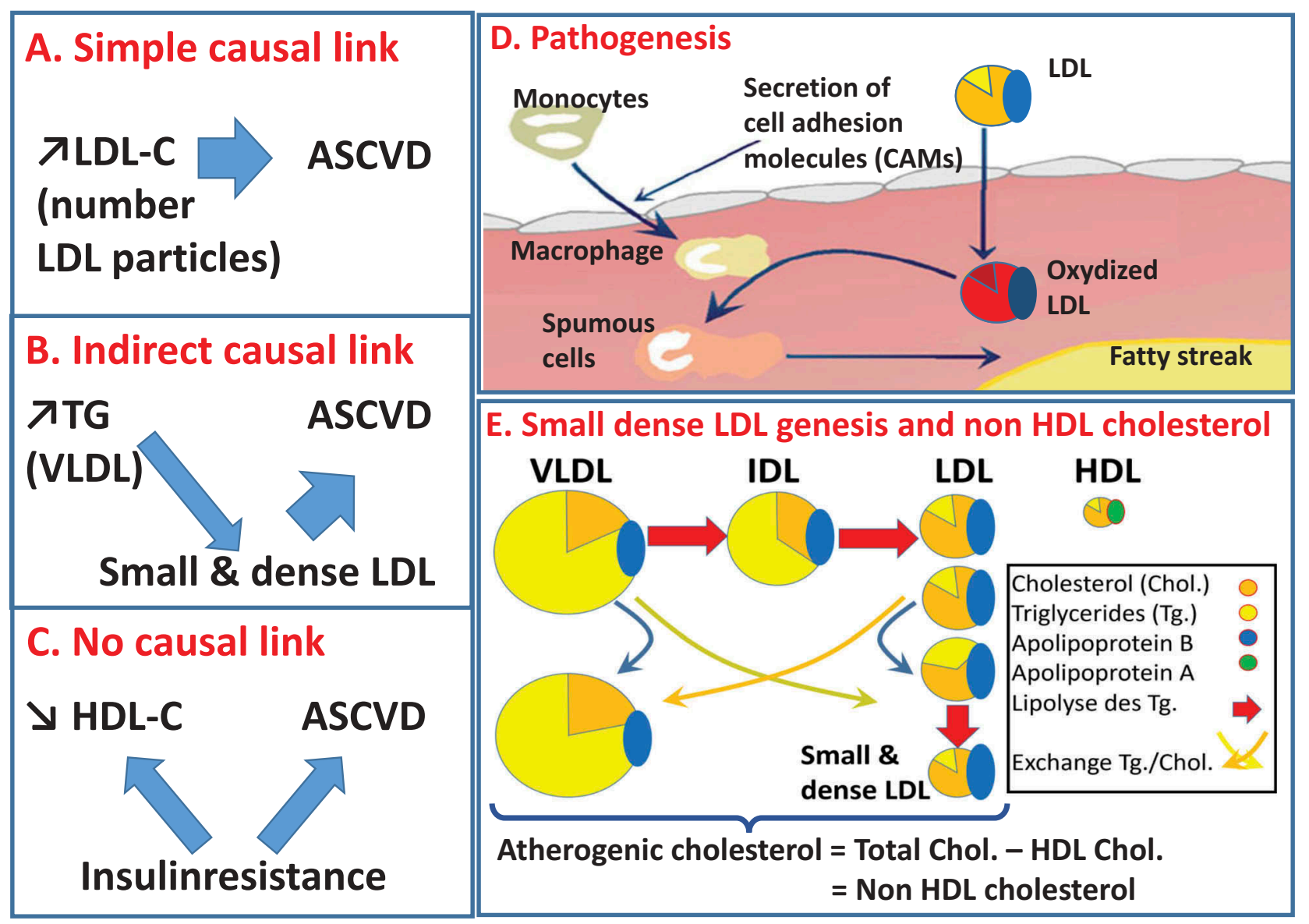

Figure 1. Causal relationship between lipid parameters and atherosclerotic cardiovascular disease (ASCVD).

The panels A-C show the different relationships between the common lipids parameters and ASCVD. The panel $D$ is a scheme of the pathogenesis of atherosclerosis where the first step is the retention in the artery wall of LDL (and other apoB-containing lipoproteins) followed by the cascade of oxidation, macrophages and lymphocytes migration, inflammation, smooth cell proliferation that cause atherosclerosis, and later on arterial stenosis, plaque disruption and embolization leading to acute or chronic coronary, cerebrovascular or peripheral ischaemia. Panel E. Genesis of small dense LDL: the presence of high level of triglycerides (Tg.) is associated to high levels of Tg-rich VLDL which can undergo transfer (through the enzymatic activity of CETP) of their Tg to LDL (less rich in TG) in exchange of the cholesterol of LDL (richer in cholesterol) to VLDL (poor in cholesterol). The hydrolysis of Tg (i.e. by hepatic lipase) in these Tg-enriched /cholesterol-depleted LDL, gives rise to small dense LDL.

on the idea that there is indeed a causal link between LDL particles and ASCVD and that the reduction of LDL is effective in significantly reducing the risk of ASCVD. That results in proclaiming LDL-C level as a primary target for ASCVD prevention.

In contrast, the same approaches (intervention trials with drugs specifically able to increase HDL cholesterol (HDL-C) and Mendelian randomization genetic studies) were not able to show a causal link between HDL-C and ASCVD [4]. While remaining a 'marker' of risk (because it helps to estimate the total cardiovascular risk), the HDL-C level is not targeted in prevention strategies. Regarding triglycerides, the association with ASCVD exists but no target is set because the association becomes null after adjustment for non-HDL-cholesterol (non-HDL-C) which is an estimate of the concentration of all apo B-containing lipoproteins (Figure 1).

\section{There is no lower limit for LDL cholesterol}

A question that arises from the causal association of LDL with ASCVD is 'how low can we get for LDL-C and is there an LDL-C threshold for clinical benefit?'. The LDL-C limit (also called 'target') recommended in the past guidelines has evolved quite a lot over time. A quarter of a century ago, the $4 \mathrm{~S}$ trial was the first modern trial on lipid-based ASCVD prevention in patients with established coronary disease [5]. These patients had high levels of LDL-C (around $190 \mathrm{mg} / \mathrm{dL}$ ) and the reduction of LDL-C to the normal range (around $120 \mathrm{mg} / \mathrm{dL}$ ) was associated with a significant benefit in terms of all-cause mortality and major coronary event. This allowed to conclude that 'high is bad' and that a reduction of high levels with simvastatin was effective and safe. The next question was then: "Should we also lower LDL-C in individuals with socalled average levels of LDL-C?" The idea that average ('normal' in terms of population distribution) is not 'appropriate' for the arterial wall was a tricky concept, different from what we know from many biological variables where the average level in the general population is the ideal level for health. A series of trials like 'CARE' [6] and 'the Heart Protection Study' [7] were thus carried out in individuals in secondary prevention 
with average levels of LDL-C (around 120-150 mg/dL). The significant benefit demonstrated in achieving LDL$C$ less than $100 \mathrm{mg} / \mathrm{dl}$ in these patients demonstrated that "average is not good" and supported the new target of $<115 \mathrm{mg} / \mathrm{dL}$ in the 1998 European guidelines [8] then $<100 \mathrm{mg} / \mathrm{dl}$ in the 2003 European guidelines [9].

Subsequent trials like 'PROVE IT' [10] and 'TNT' [11] comparing high-intensity statin therapy (i.e. atorvastatin $80 \mathrm{mg}$ ) with low-intensity therapy (i.e. pravastatin $40 \mathrm{mg}$ ) have showed undoubtfully that achieving LDL$\mathrm{C}$ to $<70 \mathrm{mg} / \mathrm{dL}$ is further associated with a reduction in ASCVD events. Meanwhile, the Cholesterol Treatment Trialists (CTT) Collaboration meta-analysis demonstrated that the ASCVD benefit is linearly correlated with the reduction of LDL-C so that this could be expressed by a 'rule': each reduction of LDL-C by $40 \mathrm{mg} / \mathrm{dL}$ is associated with a relative reduction in ASCVD events of $20 \%$ [3]. The message becomes thus 'Lower is better' or 'Even more intensive LDL lowering is better' which supported the concept of highintensity statin therapy. So, in 2011, lipid guidelines issued an update recommending LDL-C target at $<70 \mathrm{mg} / \mathrm{dL}$ in patients at very high total CV risk [12] and this remained at that level in the 2016 guidelines [13].

Then, in 2016, were published the results from the 'IMPROVE IT' trial where LDL-C was further lowered by adding ezetimibe on top of a statin in patients with established ASCVD in whom the LDL-C was $>70 \mathrm{mg} / \mathrm{dL}$ [14]. With this combination an LDL-C around $55 \mathrm{mg} / \mathrm{dL}$ was achieved and this resulted in more ASCVD prevention suggesting that 'even lower is even better' and that combining statin therapy with a cholesterol absorption inhibitor is beneficial and safe.

That has set the rationale for an LDL-C target even lower than $<70 \mathrm{mg} / \mathrm{dL}$. This has been tested in the most recent clinical trials, 'FOURIER' and 'ODYSSEY OUTCOMES' with the monoclonal antibodies against PCSK9 given in very high-risk patients with LDL-C around $70 \mathrm{mg} / \mathrm{dL}$ despite optimally treated with statin $[15,16]$. The further reduction of LDL-C to a very low level (40 mg/dL and less) resulted in $15-20 \%$ reduction in major CV events. Importantly, sub-analyses of these trials supported the hypothesis that there appeared to be no LDL-C threshold for clinical benefit and no risk related to achieving 'very very low' levels. A FOURIER sub-analysis [17] that examined the results in terms of achieved LDL-C demonstrated a monotonic nearly linear relationship between achieved LDL-C and the risk of major CV events (CVD death, MI or stroke) till levels as low as $20 \mathrm{mg} / \mathrm{dL}$. Interestingly the shape of this linear curve is similar to the relationship between the achieved LDL-C and the change of the coronary plaque volume measured by IVUS in the GLAGOV trial [18] where patients were randomized to receive PCSK9 inhibitors versus placebo on top of a statin. Results from the GLAGOV trial confirmed a critical LDL-C level (between 70 and $90 \mathrm{mg} / \mathrm{dL}$ ), already pointed out in previous IVUS trials [19], where the plaques grow if the achieved LDL-C is above that level and plaques regress if below that level. The similarity between the curves underpins the mechanistic process behind prevention: to prevent a ASCVD event, plaques need to shrink, and that can be achieved by lowering LDL-C below 70-90 mg/dL. Another FOURIER sub-analysis [20] examined the subgroup of patients with baseline LDL-C less than $70 \mathrm{mg} / \mathrm{dL}$ ( $\mathrm{N}=$ 2034; median 66 mg/ $\mathrm{dL})$, a level where according to previous guidelines, it was not clear to reduce that level further. Under PCSK9 inhibitors, they achieved a median LDL-C of $21 \mathrm{mg} / \mathrm{dL}$ (IQR 11,5-37, which means that a quarter had LDL-C below $11,5 \mathrm{mg} / \mathrm{dL}$ ) and the relative benefit was just as great as in those who had higher levels of LDL-C to start with .

So, this clarifies the idea that we should treat the patients at very high total ASCVD risk with the rule 'Lowest is better'. That was confirmed in a more recent meta-analysis of the subgroups of patient starting with LDL-C levels averaging $70 \mathrm{mg} / \mathrm{dL}$ or less in various statin and non-statin trials [21]: the risk of major vascular events is significantly reduced by $21 \%$ for $38.7 \mathrm{mg} / \mathrm{dL}$ (after adjustment) reduction in LDL-C, which is virtually of the same magnitude as what was observed in the Cholesterol Treatment Trialists Collaboration analysis in which the starting LDL-C was nearly twice as high.

\section{Treat earlier}

Another question that arises from the causal association of LDL with ASCVD is 'What if we lower LDL-C earlier in life?'. Knowledge of the impact of lifelong exposure to lower LDL-C levels on future risk for ASCVD has been driven largely by insights from Mendelian randomisation studies [22-24]. Individuals with genetic variants lowering even slightly LDL-C levels compared to the general population have a strong lifelong CV risk reduction. This may suggest that treating more persons earlier in life with even less intensive therapy could ultimately lead to more prevention of ASCVD at the population level [25].

The concept of lifelong cholesterol burden is a little bit like smoking where we think about 'pack years': someone who had smoked 2 packs a day for 20 years has a 40 pack-year smoking history. The same concept can apply for LDL-C. This is illustrated in Figure 2, where the $\mathrm{X}$-axis is the lifetime, the $\mathrm{Y}$-axis their cumulative amount of LDL-C. The slope of the line is simply a function of the circulating level of LDL-C. With an average level of LDL-C (around $120 \mathrm{mg} / \mathrm{dL}$ ), the line will reach at age 70 the red line which is the threshold for ASCVD (arbitrarily drawn here, as it will vary from person to person depending on other risk factors 


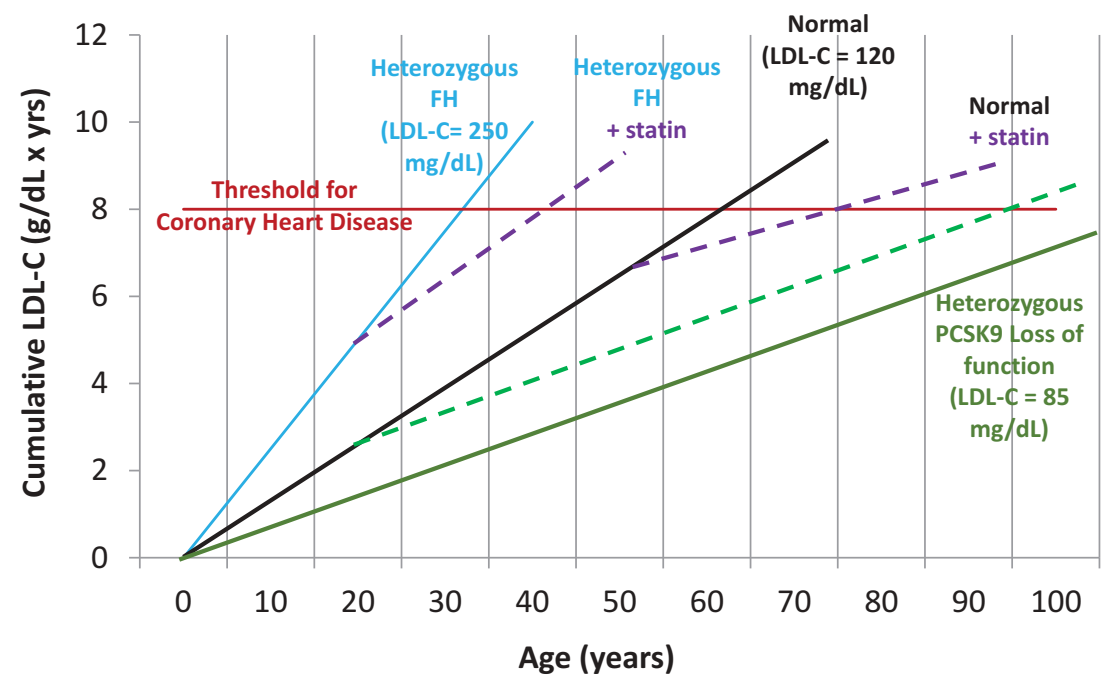

Figure 2. Effect of lifelong exposure of LDL cholesterol and the effect of LDL lowering therapy at various ages.

predisposing to ASCVD such as hypertension, diabetes and smoking). Some people are genetically at risk, for example, those having heterozygous familial hypercholesterolemia ( $\mathrm{HeFH}$, with a steeper blue line) with $\mathrm{LDL}-\mathrm{C}$ levels around $250 \mathrm{mg} / \mathrm{dL}$ (or worse for the rare homozygous $\mathrm{FH}$ ) and they will get to the threshold for ASCVD earlier so they'll develop clinical events prematurely. Others are genetically protected, for example, those who have a PCSK9 loss of function variant at the heterozygous state (with a flatter green line) with LDLC levels around $85 \mathrm{mg} / \mathrm{dL}$ and they will be protected from developing ASCVD. There is little debate about treating people with $\mathrm{HeFH}$ early as they are at very high risk and as there is evidence that treating them early, will delay the onset of ASCVD and thus shift their curve to the right. What about average individuals? Typically, our guidelines have been tethered to the notion of 10-year risk and because age is the ultimate driver for risk, it's very hard to have a high 10-year risk until one is in its mid 50s. That means however that we are neglecting all the time that arteries may be exposed to high levels of LDL-C, leading to subclinical atherosclerosis. If we start treating in the $50 \mathrm{~s}$, it is very hard to bend the curve in Figure 2. What would happen if we treat them earlier (in the 20s) and reduce their LDL-C by $40 \%$ ? Doing this, the slope of their curve can really be changed (the dotted green line); in other words, they should be greatly protected from coronary disease as it happens in the carriers of loss of function PCSK9 variant who have a $80 \%$ lifelong reduction of ASCVD with a delay of onset to about 30 years. This is further supported in a recent observational study [26] comparing coronary artery calcium (CAC) in the MultiEthnic Study of Atherosclerosis (MESA) population in the United States (where average LDL-C is around $120 \mathrm{mg} / \mathrm{dL}$ ) with the "Tsimane population" (a preindustrial society in the Bolivian Amazon who have an average LDL-C of $75 \mathrm{mg} / \mathrm{dL}$ ). The $40 \% \mathrm{LDL}$ cholesterol reduction between these two populations ( 75 versus
$120 \mathrm{mg} / \mathrm{dL}$ ) shifts the burden of atherosclerosis by approximately 30 years.

\section{The end of some misconceptions}

It is interesting to point out how these recent findings have explicitly rejected a number of concepts that have been promoted in the past and are sometimes subjected to polemics.

The first misconception is that HDL cholesterol is the good cholesterol and LDL cholesterol is the bad one. A low HDL-C level indicates the presence of other underlying ASCVD risk factors like insulin-resistance, lack of physical activity, excess of blood triglycerides or others. As it somehow quantifies these underlying disorders, HDL-C remains a good risk marker for the estimation of the total CVD risk. However, drug therapies that increased the HDL-C levels failed to demonstrate risk reduction. Therefore, $\mathrm{HDL}-\mathrm{C}$ is not a target for therapy and treatment should therefore focus on correcting the underlying cause of low HDL-C.

LDL but also other apoB-containing lipoproteins (VLDL and IDL) causes atherosclerosis. So, the new 2019 ESC/EAS guidelines emphasizes the use of the level of apolipoprotein B or, if not available, the 'nonHDL-C level' as secondary targets. The 'non-HDL-C' level takes into account the cholesterol of all of the atherogenic apoB-containing lipoproteins: LDL, small dense LDL, IDL and VLDL and lipoprotein(a) (Figure 1). It appears to be more stable over time than triglyceride levels in practice and is well correlated with the incidence of ASCVD. It is thus used as secondary target (or even primary target if triglycerides exceed $400 \mathrm{mg} / \mathrm{dL}$ rendering the LDL-C countless by the Friedewald formula) especially in patients with elevated triglycerides, with metabolic syndrome or with diabetes [27].

The second misconception is that the level of LDL-C (or apoB or non-HDL-C) does not improve risk prediction. It is now well established that LDL and other 
apoB-containing lipoproteins cause ASCVD. However, adding LDL to a risk prediction model does not improve its discrimination. This is largely an artifact of a mathematical dominance of age in most of the current risk models. Indeed, one could add any risk factor (smoking, blood pressure, calcium score or polygenic risk score with hazard ratio more than one) and none of those would improve the discrimination because of this mathematical dominance of age. So to overcome this dominance of age, the new 2019 ESC/EAS guidelines specifically suggest a nuanced approach for risk assessment especially for younger and older individuals (see below).

The third misconception is that statins are somehow magical and reduce risk by doing other things (the socalled 'pleiotropic effect') than lowering LDL-C. In the trials, if we plot the PCSK9 trials or the ezetimibe trial on the cholesterol treatment trials regression line (eventually redefined by duration of follow up), the points are exactly on the same line whatever the drugs used to lower LDL-C. In Mendelian randomization studies, we see that the variances in the HMG Co reductase gene, in the PCSK9 gene and in the NPC1L1 gene that mimic respectively statins, PCSK9 inhibitors and ezetimibe are associated with the same reduction in the risk of ASCVD per any change in LDL-C. So, the new 2019 ESC/EAS guidelines explicitly reject the mythology of statins by suggesting that we should move away from the recommendation of high-intensity statins towards high-intensity lipidlowering therapy.

The fourth misconception is that the benefit of LDL lowering therapy (statins in particular) is largely independent of baseline LDL levels. That is a misconception based on the past idea that it is the percent reduction in LDL-C that drives the benefit. The cholesterol treatment trial (CTT) regression line shows that the clinical benefit is in reality determined by the absolute reduction in LDL-C, the same relative risk reduction is produced from the same

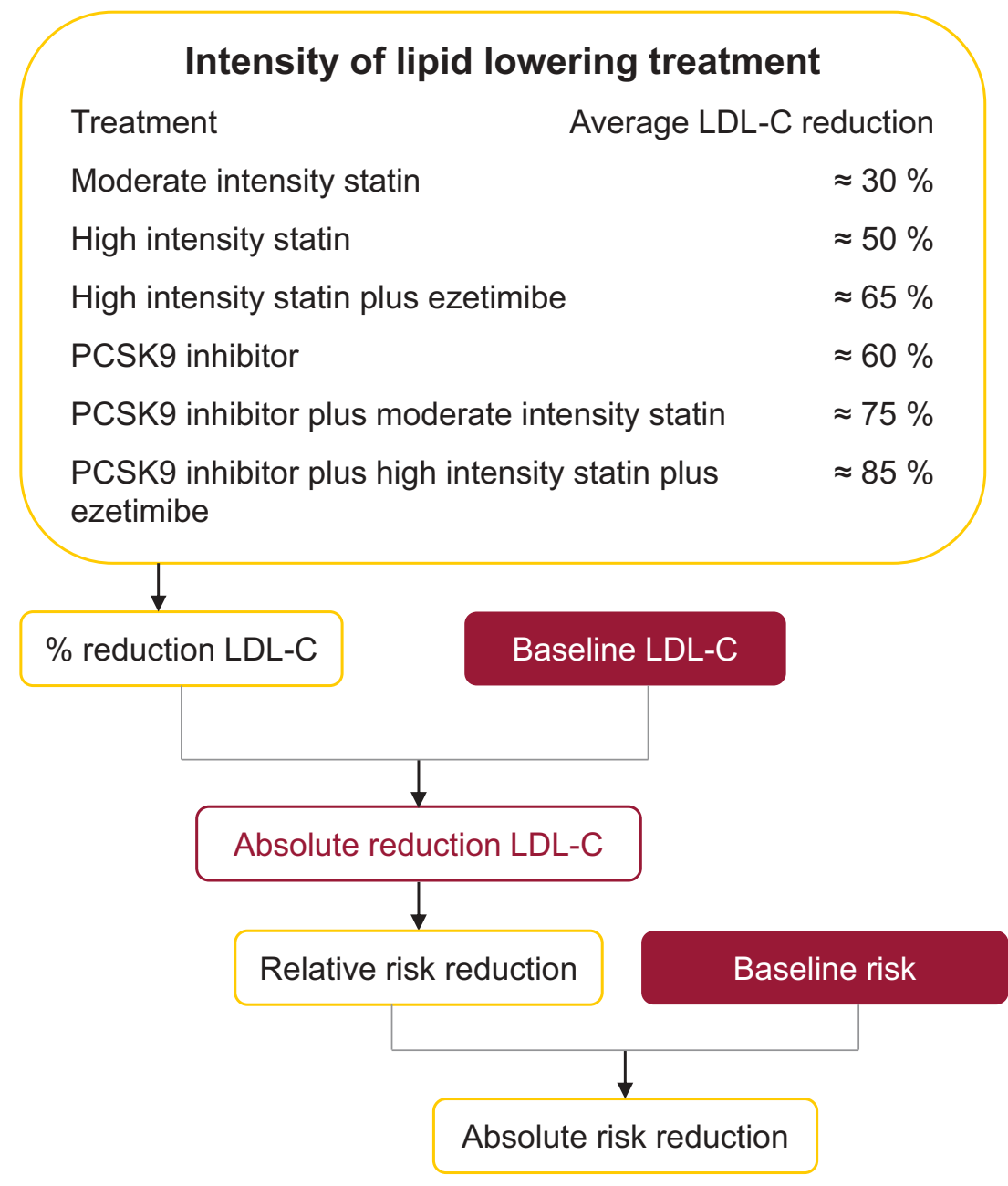

Figure 3. How to calculate the benefit from a lipid-lowering intervention.

The guidelines declare that the benefit on lipid-lowering therapy is directly proportional to the absolute change in LDL and other apoB-containing lipoproteins but that this absolute change must be interpreted in the context of somebody's baseline level of risk. Indeed, the intensity of lipid-lowering therapy whether it's a statin, ezetimibe or PCSK9 inhibitors or some combination determines an expected percentage of reduction of LDL. That percentage of reduction of LDL-C multiplied by the baseline LDL-cholesterol level determines the absolute reduction in LDL cholesterol. As shown by the cholesterol treatment trial data, it is the absolute reduction of LDL-C that determines the expected clinical benefit expressed in relative risk reduction with the following rule: $20 \%$ reduction in CV events per $40 \mathrm{mg} / \mathrm{dL}$ LDL-C. We can thus multiply the relative risk reduction calculated from this rule by the baseline risk in order to estimate the expected absolute risk reduction from any lipid-lowering therapy (adapted from Mach et al. ESC/EAS guidelines for the management of dyslipidaemias: lipid modification to reduce cardiovascular risk. Eur Heart J. 2019 Aug 31. pii: ehz455. doi: 10.1093/eurheartj/ehz455). 
absolute reduction in LDL-C regardless of the baseline LDL-C. So, the new 2019 ESC/EAS guidelines now propose a simple algorithm in order to anticipate the expected clinical benefit of any lipid-lowering therapy based on the intensity of therapy and the baseline LDL-C and the baseline risk (Figure 3). As we can extrapolate from this algorithm, the higher the patients baseline risk and/or the greater the LDL reduction, the fewer patients you need to treat to prevent one event (NNT).

\section{What is new in the 2019 ESC/EAS guidelines}

The fundamental concepts summarized above have formed the basis of the new 2019 ESC/EAS guidelines [28]. Guidelines establish paradigms based on the existing evidence and then scientists test and extend those paradigms to determine whether they are correct and determine where we need to go next. And then as we go through this cycle, the guidelines are revised and updated based on the available evidence. For the ASCVD prevention guidelines, we've seen progressively a shift in the paradigms on how to reduce CV risk, moving from just simple risk factor level-based treatments, to global CV risk assessment-based approaches and now, as for the new-released 2019 ESC/EAS lipid guidelines [28], increasingly thinking more about benefit-based approaches with the notion of maximising expected benefit rather than simply treating the risks.

To do so, the guidelines recommend to start with a comprehensive assessment of the total risk of developing ASCVD in order to titrate the intensity of the prevention strategy in accordance with the baseline LDL-C level and the baseline total risk of ASCVD. The guidelines prioritize the identification of people at high or very high 10-year risk of experiencing a fatal cardiovascular event because they are most likely to derive the greatest short-term clinical benefit from aggressively lipid lowering therapy; but recommendations also emphasize the need to pay attention to intermediate and low-risk subjects, especially those in which risk modifiers will mitigate the risk to a higher lifelong ASCVD risk.

\section{Comprehensive assessment of ASCVD risk and classification in four risk categories}

The guidelines recommend that we begin with a comprehensive assessment of ASCVD risk which proceed in three stages (all at the IC level of class of recommendation and level of evidence).

1. Risk assessment begins with a thorough clinical evaluation to identify those clinical situations that are associated with a high short-term risk of events.

The very high-risk category includes patients who have documented ASCVD either clinical or unequivocal on imaging. These are patients with a personal history of an acute coronary syndrome, stable angina, coronary revascularization, stroke or TIA and peripheral arterial disease and patients in whom a significant plaque has been found in the coronaries or in the carotid arteries. In addition, other clinical characteristics that define a very high-risk patient are diabetes (PLUS end organ damage or 3 or more risk factors or early onset and long duration of diabetes), severe chronic kidney disease (CKD) (defined as an estimated GFR $<30 \mathrm{ml}$ / $\mathrm{min} / 1.73 \mathrm{~m}^{2}$ ), familial hypercholesterolemia (PLUS either ASCVD or another major risk factor).

The high-risk category includes patients who have markedly elevated single risk factor levels (most notably total cholesterol $>310 \mathrm{mg} / \mathrm{dL}, \mathrm{LDL}-\mathrm{C}>190 \mathrm{mg} / \mathrm{dL}$ or blood pressure $>180 / 110 \mathrm{mmHg}$ ). Other clinical characteristics include familial hypercholesterolemia (but without other risk factors), diabetes (without end organ damage, with duration of diabetes $\geq 10$ years or with another additional risk factor) or moderate CKD (30 to $60 \mathrm{ml} / \mathrm{min} / 1.73 \mathrm{~m}^{2}$ ).

The clinical characteristics that define persons who are at moderate risk are younger patients with diabetes $(<35$ yrs with type 1 diabetes or $<50$ yrs with type 2 diabetes) with duration of diabetes less than 10 years but no other major risk factors.

2. Next, if the patients do not have these clinical characteristics, the recommendation is to use the SCORE model to estimate the 10-year risk of fatal CVD. The SCORE algorithm is an extremely intuitive and easy to understand metric that is presented in men and women separately at different ages and focuses on the three most important modifiable causes of atherosclerosis: LDL and apoB-containing lipoproteins estimated by total cholesterol, systolic blood pressure and smoking. The SCORE allows to classify the individuals into the four categories of very high risk (calculated SCORE for 10-year risk of fatal CVD $\geq$ $10 \%)$, high risk ( $\geq 5 \%)$, moderate risk $(\geq 1 \%)$ and low risk $(<1 \%)$. The SCORE charts that are now presented in the 2019 guidelines differ slightly from previous ones because now interactions between age and the associations between the major risk factors and ASCVD have been taken into account; the new charts also have results for persons aged $70+$.

3. Finally, it is recommended to refine and individualise the risk estimation by taking into account 'risk modifiers' that are not captured in the risk algorithms. This is particularly true for persons who are at moderate risk according to SCORE. In those individuals, the consideration of risk modifiers may move up or down the total CVD risk estimate (and thus the risk category) and this will influence the choice of the preventive approach. One of these modifiers, particularly in women, is HDL-C level. Currently in Belgium, we are using the SCORE recalibrated on Belgian epidemiological data [29] and we can use multipliers to nuance the 10-year risk estimate as a function of HDL- 
Table 1. Comparison of the LDL-C treatment goals in the subgroups of total CV risk in the ESC/EAS guidelines from 2016 to 2019 (adapted from Mach et al. ESC/EAS guidelines for the management of dyslipidaemias: lipid modification to reduce cardiovascular risk. Eur Heart J. 2019 Aug 31. pii: ehz455. doi: 10.1093/eurheartj/ehz455).

Comparison of the LDL-C goals in the ESC/EAS Guidelines 2016 and 2019

\begin{tabular}{|c|c|c|}
\hline \multirow[b]{2}{*}{ Risk category } & \multicolumn{2}{|c|}{ LDL-C goals } \\
\hline & 2016 & 2019 \\
\hline Very-high-risk & $\begin{array}{c}<70 \mathrm{mg} / \mathrm{dL} \\
\text { or }>50 \% \downarrow \downarrow^{\mathrm{a}} \text { if baseline LDL-C } 70-135 \mathrm{mg} / \mathrm{dL}\end{array}$ & $\begin{array}{l}<55 \mathrm{mg} / \mathrm{dL} \\
\text { and }>50 \% \downarrow \text { a }\end{array}$ \\
\hline High-risk & $\begin{array}{c}<100 \mathrm{mg} / \mathrm{dL} \\
\text { or }>50 \% \downarrow^{\mathrm{a}} \text { if baseline LDL-C } 100-200 \mathrm{mg} / \mathrm{dL}\end{array}$ & $\begin{array}{l}<70 \mathrm{mg} / \mathrm{dL} \\
\text { and }>50 \% \downarrow \text { a }\end{array}$ \\
\hline Moderate-risk & $<115 \mathrm{mg} / \mathrm{dL}$ & $<100 \mathrm{mg} / \mathrm{dL}$ \\
\hline Low-risk & $<115 \mathrm{mg} / \mathrm{dL}$ & $<115 \mathrm{mg} / \mathrm{dL}$ \\
\hline
\end{tabular}

${ }^{a}$ Reduction from the untreated baseline LDL-C level.

C level [30]. For example, a 60-year-old woman who is a smoker, has SBP of $140 \mathrm{mmHg}$ and total cholesterol of $230 \mathrm{mg} / \mathrm{dL}$ may have an estimated 10-year risk varying from 2 to 10 , thus a moderate risk to a very high risk, if her $\mathrm{HDL}$ cholesterol varies between 80 and $20 \mathrm{mg} / \mathrm{dL}$. The other most important of those risk modifiers is a family history of premature atherosclerosis; others that should be considered are triglyceride levels, $L p(a)$, social deprivation, lack of physical activity, obesity and central obesity, chronic immunemediated inflammatory disorders, major psychiatric disorders, HIV treatment, obstructive sleep apnea and non-alcoholic fatty liver disease. And then if uncertainty remains especially in persons at moderate risk according to SCORE, it is recommended to search for subclinical atherosclerosis using non-invasive CV imaging. Coronary artery calcification (CAC) scoring is clearly the best indicator of making better decisions. And that happens because half people with a moderate risk have a CAC score of 0 which reclassifies them to a much lower risk estimate. As shown in the study of Nazir et al. [31], people with moderate risk but a CAC score of zero don't achieve the benefit threshold (benefit over harm) over 10 years of followup whereas people above CAC score of 100 clearly do. So the specific recommendation is to defer therapy in patients with the CAC score of 0 .

It's important to recognise that risk estimating equations have a number of limitations. The most important is that it is mathematically dominated by age. Importantly age is not a risk factor for ASCVD but is merely a metric of the cumulative exposure to lifetime burden of those factors that cause atherosclerosis.

The SCORE model almost uniformly underestimates the risk of cardiovascular disease in younger patients. A 40-year-old man even if he smokes, if he has a very long lifetime exposure of causal LDL and an elevated blood pressure will be classified at low or moderate risk of ASCVD. In these young patients, to contextualize that information can be done by explaining to the patient that the short-term risks may be fairly low based on the equation, but, simply by virtue of their risk factor combination, that he has first a high 'relative risk' compared with other people of his age and that he has secondly the same risk as a 65-year-old man with ideal risk factors (non-smoker, total cholesterol $<160 \mathrm{mg} / \mathrm{dL}$ and SBP < $120 \mathrm{mmHg}$ ). This so-called 'risk age concept' may be a particularly motivating metric to change lifestyle.

Due to the mathematical dominance of age within all risk estimating equations, nearly all men and women above 70 years old are classified as high or very high risk. Because of the aggressive goals for LDL reduction among people with high and very high risk, it is thus particularly important to contextualise this information by considering other risk modifiers. For example, atherosclerotic imaging may identify those people who may be less vulnerable or have had low lifetime exposures to the combination of LDL, SBP and smoking.

\section{New LDL-cholesterol target in all risk categories}

The new guidelines reinforce the idea that LDL-C levels should be lowered as much as possible to prevent ASCVD, especially in high and very high-risk patients (Table 1).

\section{High and very high-risk patients}

Very high-risk patients (in both primary and secondary prevention) should achieve an LDL-C level of $<55 \mathrm{mg} /$ $\mathrm{dL}$ and at least a $50 \%$ reduction from the baseline $\mathrm{LDL}-$ $C$ level, high-risk patients an LDL-C level of $<70 \mathrm{mg} / \mathrm{dL}$ and at least a $50 \%$ reduction from the baseline $\mathrm{LDL}_{\mathrm{C}} \mathrm{C}$ level. Compared to the last guidelines the wording 'OR' had been replaced by 'AND' to make clear that what counts is the amplitude of reduction ('Lower is better') as well as the achievement of the lowest target as possible ('Lowest is better'). Results from subgroup analyses of trials with ezetimibe [32] and with PCSK9 inhibitors [33-37] also demonstrate that amongst patients with extremely elevated ASCVD risk an even lower LDL-C goal may be considered. This has resulted in a recommendation (class Ilb) that for patients with ASCVD who experience a second vascular event within 2 years (not necessarily of the same type as the first event) while taking maximally tolerated statin-based 
therapy, an LDL-C goal of $<40 \mathrm{mg} / \mathrm{dL}$ may be considered.

To achieve such low LDL-C goals, the guidelines emphasize the importance of combination treatment, combining high-intensity statins with ezetimibe. If the LDL-C goal is not achieved after 4-6 weeks despite maximally tolerated statin therapy and ezetimibe, a PCSK9 inhibitor is recommended.

\section{Low- and intermediate-risk people}

In these people, the ESC/EAS guidelines recommend reinforcing the lifestyle change and suggest the use of lipid-lowering drugs to lower LDL-C in those with other qualifiers that put them at a higher total CVD risk (see above). It is important to note that the new LDL-C target of $<100 \mathrm{mg} / \mathrm{dl}$ in intermediate-risk individuals can be a difficult target to achieve with lifestyle alone taking into account the average Belgian LDL-C of $130 \mathrm{mg} / \mathrm{dl}$. Furthermore, the recommendation for lipid-lowering drugs in the intermediate-risk group has been upgraded from 'could be considered' to 'should be considered'. Whilst this is a very large group of individuals in which lipid-lowering therapy should now be considered, this is scientifically sound and reflective of the potential benefits at the population level.

\section{Treatment of dyslipidaemia in older patients}

Treatment with statins is recommended for primary prevention, according to the level of risk, in older people aged $\leq 75$. A statin may be considered for primary prevention in older people aged $>75$. Particularly in these older patients, statin treatment should be started at a low dose specially if there is significant renal impairment and/or if there is potential for drug interactions, and then titrated upwards to achieve the LDL-C treatment goal.

In a recent publication from the $\mathrm{C} T \mathrm{~T}$ collaboration, it is shown that in the 65-70+ years old, the patients should keep their statin if there was an earlier indication [38].

\section{News on other lipid parameters: triglycerides and lipoprotein(a)}

While statin treatment remains the first choice for managing high triglycerides ( $>200 \mathrm{mg} / \mathrm{dL}$ ), n-3 PUFAs (particularly icosapent ethyl $2 \times 2 \mathrm{~g}$ daily) 'should be' considered in high-risk patients with persistently elevated triglycerides (between 135 and $499 \mathrm{mg} / \mathrm{dL}$ ) despite statin treatment. The new recommendations of $n-3$ PUFAs are supported by the evidence from REDUCE-IT trial [39].

Fenofibrate (or bezafibrate) may be considered in combination with statins in high-risk patients who are at LDL-C goal but with persistently elevated triglycerides $>200 \mathrm{mg} / \mathrm{dL}$.
In people with high triglycerides, diabetes, obesity or very low (either naturally occurring or therapeutically achieved) LDL levels, the guidelines also recommend the use of non-HDL-C because it is a derived calculated metric which is available with a standard lipid profile at no cost and it is an estimate of the cholesterol content of all apoB-containing lipoproteins that cause atherosclerosis. There is also a new recommendation that apoB should be measured for assessing the clinical benefit of any lipid-lowering therapy as it is directly proportional to the absolute change in the apoB-containing lipoprotein concentration. NonHDL-C or apoB can be used as an alternative to LDL-C when LDL-C cannot be calculated (triglycerides $>400 \mathrm{mg} / \mathrm{dL}$ ).

Also new to these guidelines is the recommendation that lipoprotein(a) [Lp(a)] should be measured at least once in each person's lifetime. Epidemiologic and Mendelian randomisation studies support that $\operatorname{Lp}(a)$ is causal in ischaemic heart disease $[40,41]$ and thus a risk modifier that nuances the ASCVD risk in low,moderate and high-risk individuals. Specifically, persons who have extreme elevations of $L p(a)$ above $180 \mathrm{mg} / \mathrm{dL}$ (or $>430 \mathrm{nmol} / \mathrm{L}$ ) may have a lifetime risk of ASCVD that is equivalent to that observed in patients with $\mathrm{FH}$. This situation that is twofold more prevalent than $\mathrm{FH}$ may represent a new inherited lipoprotein ASCVD risk disorder. The new emphasis on $L p(a)$ is important, given the fact that novel treatments that are specific to this lipoprotein abnormality are now entering phase III clinical trials in high and very high-risk patients [42]. Currently, treatment of high $L p(a)$ is limited to the PCSK9 inhibitors which have been shown to reduce levels by $25-30 \%$ on average $[43,44]$.

\section{Familial hypercholesterolaemia}

Familial hypercholesterolaemia (FH) is an autosomal dominant lipoprotein disorder characterized by significant elevation of LDL-C and markedly increased risk of premature ASCVD. The prevalence of $\mathrm{FH}$ is $1 / 300$ (around 40.000 Belgians). Familial hypercholesterolaemia $(\mathrm{FH})$ remains under-diagnosed and under-treated worldwide. Up to 2013 in Belgium, only a fraction of heterozygous FH carriers have been genetically characterized [45]. Awareness of this disease is thus crucial for recognizing $\mathrm{FH}$ amongst all patients visiting their doctors or admitted for ASCVD events in hospitals. Once identified, extended cascade screening in the family is the best approach to identify and treat as early as possible (around age 10 years). In Belgium, several initiatives have been launched these last 5 years to increase the efficiency of diagnosis such as the creation of a patient association for FH (www. Belchol.be) which organized several awareness campaigns and a parliament conference, a consensus paper on the management of $\mathrm{FH}$ in children [46], the 
organization of a strategy to facilitate the detection and management of $\mathrm{FH}$ among patients hospitalized for an ASCVD event in more than 35 coronary care units [47] and an ongoing study examining the feasibility of cascade screening with the collaboration of field nurses in our country.

\section{Side effects and adherence of lipid-lowering drugs}

During the last years, side effects of lipid-lowering therapy have been overemphasized by the media and fake news about 'cholesterol' has contributed to a poor adherence to therapy [48]. Roughly $10 \%$ of cardiovascular disease events in Europe could be attributed to poor adherence and currently, the highest poor adherence is for the statin class [49]. This could become worse because by setting up lower LDL-C goals the adherence with lifestyle changes and drug therapy may become even more problematic.

Statins like any other drugs produce side effects but the most relevant are myalgia and diabetes. However, there have been reports proclaiming that statins induce Parkinson or Alzheimer disease or whatever other diseases. These are not based on scientific evidence, cause confusion among the public and must be contradicted [50]. Consensus panels of the EAS have summarized the problems related to side-effects of statins and how to handle these [48,51].

A low or very low LDL-C level is not dangerous. Cholesterol is the precursor of vitamin $D$, corticosteroids and gonadal steroids but their metabolism is not impaired by therapeutic lowering to very low LDL-C levels. The fact is that an organism does not need circulating cholesterol in order to function. There are examples of animals (cholesterol as low as $10 \mathrm{mg} / \mathrm{dL}$ in hippopotamus), of human populations (Tsimani), of individuals with inherited extremely low cholesterol (cholesterol as low as $10 \mathrm{mg} / \mathrm{dL}$ in homozygotes for loss of function PCSK9 mutation) and of human newborns (cholesterol is $50-70 \mathrm{mg} / \mathrm{dL}$ ) who develop normally despite their low level of cholesterol. Another interesting model for proof of concept is the young patients who have homozygote familial hypercholesterolemia and have no LDL receptor at all in their body. They have very high LDL cholesterol and their cells cannot catch LDL from the arteries. Nevertheless, their organs such as their breasts and their testes/ ovaries develop normally and they are not more susceptible to Parkinson disease, Alzheimer disease or cancers.

\section{Conclusions}

The concept of LDL-C as a cause of ASCVD is now clearly demonstrated. It is the basis of the update of the ESC/EAS guidelines. The current recommendations consider the targeting of therapy for maximal reduction events among those who are truly at risk while limiting the exposure to drugs in the cost in those who are not at risk. Switching from a risk-based strategy to a benefit-based approach is expected to promote substantial changes in statin eligibility especially in subjects at intermediate CV risk, modifying the subpopulation to be benefited by the treatment [52]. This is clearly the better approach for our patients and for health-care policy.

The fundamental next steps are the appropriate implementation by clinicians in their practice, together with ensuring treatment adherence by patients. Most drugs are now generic, which facilitate considerably the prescription of statin, ezetimibe and their combinations. The only limitation is for PCSK9 inhibitors which remain expensive and are not reimbursed in Belgium in all clinical situations recommended in the new ESC/EAS guidelines. So far, reimbursements are limited only to patients with familial hypercholesterolemia in addition to conventional treatments (statin and ezetimibe). Discussions within INAMI/RIZIV are underway to extend their use to other indications such as post-acute coronary syndrome if LDL-C levels are not yet sufficiently lowered under optimal treatment.

It is important that the attitude chosen to correct dyslipidemia is fully discussed with the patient. If it is up to the physician to scrupulously estimate the risks, benefits and legitimacy of treatment or non-treatment (in light of the international recommendations and local reimbursement rules) and to propose the therapeutic options, it is up to the patient to decide on the choice of treatment (or non-treatment) based on the information clearly provided by the doctor. In this regard, it is essential to discuss with them about the issues involved in each therapeutic choice, both in terms of benefits and in terms of constraints and risks of side effects. It is only with this spirit that patients will be keen to follow their treatment.

\section{Acknowledgements}

Beside the idea developed in the 2019 EAS/ESC guidelines, the present article has benefited from inspiring ideas shared during the 2019 ESC congress in Paris by professor Marc Sabatine (Distinguished chair in cardiovascular disease at the Brigham's Women hospital and professor of medicine at the Harvard Medical school and by professor Brian Ference (Professor and director of research in translational therapeutics and executive director, center for naturally randomized trials at the university of Cambridge).

\section{Disclosure statement}

HE AUTHORS HAVE RECEIVED LECTURE HONORARIA, CONSULTANCY FEES AND/OR RESEARCH FUNDING FROM ACTELION (F.D., J.D.S.), AMGEN (O.S.D., A.V., E.R.R., A.M., C.W.), Astra Zeneca (O.S.D., A.V., A.M.,), Boehringer 
Ingelheim (E.R.R., A.M.), Danone (O.S.D.), Johnson \& Johnson (A.M.), Merck Sharp \& Dome (O.S.D., A.V., E.R.R., A.M., J.D.S., C. W.), Mylan (O.S.D.), Novartis (E.R.R., A.M.), Novo-Nordisk (E.R.R., A.M.,), Eli Lilly (A.M.), Sanofi-Aventis/Regeneron (O.S.D., A.V., F. D., E.R.R., J.D.S., A.M., G.D.B, C.W.), Servier (F.D.), Teva (E.R.R.)..

\section{ORCID}

Olivier S. Descamps (D) http://orcid.org/0000-0001-9466-8295 Johan De Sutter (iD) http://orcid.org/0000-0003-1433-8152

\section{References}

[1] Ference BA, Ginsberg HN, Graham I, et al. Low-density lipoproteins cause atherosclerotic cardiovascular disease. 1. Evidence from genetic, epidemiologic, and clinical studies. A consensus statement from the European atherosclerosis society consensus panel. Eur Heart J. 2017;21(38):2459-2472.

[2] Di Angelantonio E, Gao P, Pennells L, et al. Emerging risk factors collaboration. Lipid-related markers and CVD prediction. JAMA. 2012;307:2499-2506.

[3] Baigent C, Blackwell L, Emberson J, et al. Cholesterol Treatment Trialists. Efficacy and safety of more intensive lowering of LDL cholesterol: a meta-analysis of data from 170,000 participants in 26 randomised trials. lancet. 2010;376:1670-1681.

[4] Hewing B, Moore KJ, Fisher EA. HDL and cardiovascular risk: time to call the plumber? Circ Res. 2012;111 (9):1117-1120.

[5] Scandinavian Simvastatin Survival Study Group. Randomised trial of cholesterol lowering in 4444 patients with coronary heart disease: the Scandinavian Simvastatin Survival Study (4S). Lancet. 1994;344:1383-1389.

[6] Sacks FM, Pfeffer MA, Moye LA, et al. The effect of pravastatin on coronary events after myocardial infarction in patients with average cholesterol levels. NEJM. 1996;335:1001-1009.

[7] Collins R, Armitage J, Parish S, et al. MRC/BHF heart protection study of cholesterol-lowering with simvastatin in 5963 people with diabetes: a randomized placebo-controlled trial. Lancet. 2003;361:2005-2016.

[8] Wood D, De Backer G, Faergeman O, et al. Prevention of coronary heart disease in clinical practice: recommendations of the second joint task force of European and other societies on coronary prevention. Atherosclerosis. 1998;140(2):199-270.

[9] De Backer G, Ambrosioni E, Borch-Johnsen K, et al. Third joint task force of European and other societies on cardiovascular disease prevention in clinical practice. European guidelines on cardiovascular disease prevention in clinical practice. Eur Heart J. 2003;24 (17):1601-1610.

[10] Cannon CP, Braunwald E, McCabe $\mathrm{CH}$, et al. Intensive versus moderate lipid lowering with statins after acute coronary syndromes. NEJM. 2004;350:1495-1504.

[11] LaRosa JC, Grundy SM, Waters DD, et al. Intensive lipid lowering with atorvastatin in patients with stable coronary disease. NEJM. 2005;352:1425-1435.

[12] Reiner Z, Catapano AL, De Backer G, et al. ESC Committee for Practice Guidelines (CPG) 2008-2010 and 2010-2012 committees.esc/eas guidelines for the management of dyslipidaemias: the task force for the management of dyslipidaemias of the European
Society of Cardiology (ESC) and the European atherosclerosis society (EAS). European association for cardiovascular prevention \& rehabilitation. Eur Heart J. 2011;32(14):1769-1818.

[13] Catapano AL, Graham I, De Backer G, et al. ESC scientific document group. 2016 ESC/EAS guidelines for the management of dyslipidaemias. Eur Heart J. 2016;37 (39):2999-3058.

[14] Cannon CP, Blazing MA, Giugliano RP, et al. IMPROVEIT investigators. Ezetimibe added to statin therapy after acute coronary syndromes. N Engl J Med. 2015;372(25):2387-2397.

[15] Sabatine MS, Giugliano RP, Keech AC, et al. Evolocumab and clinical outcomes in patients with cardiovascular disease. N Engl J Med. 2017;76:1713-1722.

[16] Schwartz GG, Steg PG, Szarek M, et al. Alirocumab and cardiovascular outcomes after acute coronary syndrome. N Engl J Med. 2018;379:2097-2107.

[17] Giugliano RP, Pedersen TR, Park JG, et al. FOURIER investigators. Clinical efficacy and safety of achieving very low LDL-cholesterol concentrations with the PCSK9 inhibitor evolocumab: a prespecified secondary analysis of the FOURIER trial. Lancet. 2017;390 (10106):1962-1971.

[18] Nicholls SJ, Puri R, Anderson T, et al. Effect of evolocumab on progression of coronary disease in statin-treated patients: the GLAGOV randomized clinical trial. JAMA. 2016;316(22):2373-2384.

[19] Nicholls SJ, Hsu A, Wolski K, et al. Intravascular ultrasound-derived measures of coronary atherosclerotic plaque burden and clinical outcome. J Am Coll Cardiol. 2010;55(21):2399-2407.

[20] Giugliano RP, Pedersen TR, Park JG, et al. Clinical efficacy and safety of achieving very low LDL-cholesterol concentrations with the PCSK9 inhibitor evolocumab: a prespecified secondary analysis of the FOURIER trial. Lancet. 2017;390:1962-1971.

[21] Sabatine MS, Wiviott SD, Im K, et al. Efficacy and safety of further lowering of low-density lipoprotein cholesterol in patients starting with very low levels: a meta-analysis. JAMA Cardiol. 2018;3(9):823-828.

[22] Ference BA, Robinson JG, Brook RD, et al. Variation in PCSK9 and HMGCR and risk of cardiovascular disease and diabetes. N Engl J Med. 2016;375:2144-2153.

[23] Benn M, Tybjærg-Hansen A, Nordestgaard BG. Low LDL cholesterol by PCSK9 variation reduces cardiovascular mortality. J Am Coll Cardiol. 2019;73:3102-3114.

[24] Benn M, Nordestgaard BG. From genome-wide association studies to Mendelian randomization: novel opportunities for understanding cardiovascular disease causality, pathogenesis, prevention, and treatment. Cardiovasc Res. 2018;114:1192-1208.

[25] Robinson JG, Williams KJ, Gidding S, et al. Eradicating the burden of atherosclerotic cardiovascular disease by lowering apolipoprotein B lipoproteins earlier in life. J Am Heart Assoc. 2018;7(20):e009778.

[26] Kaplan H, Thompson RC, Trumble BC, et al. Coronary atherosclerosis in indigenous South American Tsimane: a cross-sectional cohort study. Lancet. 2017;389:1730-1739.

[27] Langlois MR, Chapman MJ, Cobbaert C, et al. European Atherosclerosis Society (eAS) and the European Federation Of Clinical Chemistry And Laboratory Medicine (eflm) joint consensus initiative. quantifying atherogenic lipoproteins: current and future challenges in the era of personalized medicine and very low concentrations of LDL cholesterol. A consensus 
statement from EAS and EFLM. Clin Chem. 2018;64:1006-1033.

[28] Mach F, Baigent C, Catapano AL, et al. ESC/EAS guidelines for the management of dyslipidaemias: lipid modification to reduce cardiovascular risk. Eur Heart J. 2019. doi:10.1093/eurheartj/ehz455.

[29] De Bacquer D, De Backer G. Predictive ability of the SCORE Belgium risk chart for cardiovascular mortality. Int J Cardiol. 2010;143:385-390.

[30] Descamps OS, Cooney MT, De Backer G, et al. A simple multiplier to calculate the impact of HDL cholesterol on cardiovascular risk estimation using SCORE. Atherosclerosis. 2012;222:564-566.

[31] Nasir K, Bittencourt MS, Blaha MJ, et al. Implications of coronary artery calcium testing among statin candidates according to american college of cardiology/american heart association cholesterol management guidelines: mesa (multi-ethnic study of atherosclerosis). J Am Coll Cardiol. 2015;66(15):1657-1668.

[32] Giugliano RP, Cannon CP, Blazing MA, et al. Benefit of adding ezetimibe to statin therapy on cardiovascular outcomes and safety in patients with versus without diabetes mellitus: results from IMPROVE-IT (Improved reduction of outcomes: vytorin efficacy international trial). Circulation. 2018;137:1571-1582.

[33] Bonaca MP, Nault P, Giugliano RP, et al. Low-density lipoprotein cholesterol lowering with evolocumab and outcomes in patients with peripheral artery disease: insights from the FOURIER trial (further cardiovascular outcomes research with pcsk9 inhibition in subjects with elevated risk). Circulation. 2018;137:338-350.

[34] Sabatine MS, De Ferrari GM, Giugliano RP, et al. Clinical benefit of evolocumab by severity and extent of coronary artery disease. Circulation. 2018;138:756-766.

[35] Sabatine MS, Leiter LA, Wiviott SD, et al. Cardiovascular safety and efficacy of the PCSK9 inhibitor evolocumab in patients with and without diabetes and the effect of evolocumab on glycaemia and risk of new-onset diabetes: a prespecified analysis of the FOURIER randomised controlled trial. Lancet Diabetes Endocrinol. 2017:5:941-950.

[36] Ray KK, Colhoun HM, Szarek M, et al. Effects of alirocumab on cardiovascular and metabolic outcomes after acute coronary syndrome in patients with or without diabetes: a prespecified analysis of the ODYSSEY OUTCOMES randomised controlled trial. Lancet Diabetes Endocrinol. 2019;7:618-628.

[37] Jukema JW, Szarek M, Zijlstra LE, et al. Alirocumab in patients with polyvascular disease and recent acute coronary syndrome: ODYSSEY OUTCOMES trial. J Am Coll Cardiol. 2019;pii: S0735-1097(19)33921-X. DOI:10.1016/j. jacc.2019.03.013.

[38] Cholesterol Treatment Trialists' Collaboration. Efficacy and safety of statin therapy in older people: a meta-analysis of individual participant data from 28 randomised controlled trials. Lancet. 2019;393 (10170):407-415.

[39] Bhatt DL, Steg PG, Miller M, et al. Cardiovascular risk reduction with icosapent ethyl for hypertriglyceridemia. N Engl J Med. 2019;380:11-22.

[40] Kamstrup PR. Lipoprotein(a): the common, likely causal, yet elusive risk factor for cardiovascular disease. J Lipid Res. 2017;58:1731-1732.
[41] Tsimikas S, Fazio S, Ferdinand KC, et al. NHLBI working group recommendations to reduce lipoprotein(a)-mediated risk of cardiovascular disease and aortic stenosis. J Am Coll Cardiol. 2018;71:177-192.

[42] Tsimikas S. RNA-targeted therapeutics for lipid disorders. Curr Opin Lipidol. 2018;29:459-466.

[43] Ray KK, Vallejo-Vaz AJ, Ginsberg HN, et al. Lipoprotein(a) reductions from PCSK9 inhibition and major adverse cardiovascular events: pooled analysis of alirocumab phase 3 trials. Atherosclerosis. 2019;288:194-202.

[44] Raal FJ, Giugliano RP, Sabatine MS, et al. Reduction in lipoprotein(a) with PCSK9 monoclonal antibody evolocumab (AMG 145): a pooled analysis of more than 1,300 patients in 4 phase II trials. J Am Coll Cardiol. 2014;63:1278-1288.

[45] Nordestgaard BG, Chapman MJ, Humphries SE, et al. for the European atherosclerosis society consensus panel. Familial hypercholesterolaemia is underdiagnosed and undertreated in the general population: guidance for clinicians to prevent coronary heart disease: Consensus statement of the European atherosclerosis society. Eur Heart J. 2013;34(45):3478-3490.

[46] Descamps OS, Tenoutasse S, Stephenne $X$, et al. Management of familial hypercholesterolemia in children and young adults: consensus paper developed by a panel of lipidologists, cardiologists, paediatricians, nutritionists, gastroenterologists, general practitioners and a patient organization. Atherosclerosis. 2011;218(2):272-280.

[47] Descamps OS, Van Caenegem O, Hermans MP, et al. Belgian Atherosclerosis Society/Belgian Lipid Club (bas/blc), the Belgian Society of Cardiology (BSC) and the Royal Belgian Society of Laboratory Medicine (RBSLM). A Belgian consensus strategy to identify familial hypercholesterolaemia in the coronary care unit and its subsequent cascade screening and treatment: BEL-FaHST (the Belgium Familial Hypercholesterolaemia Strategy). Atherosclerosis. 2018;277:369-376.

[48] Mach F, Ray KK, Wiklund O, et al. European atherosclerosis society consensus panel. Adverse effects of statin therapy: perception vs. the evidence - focus on glucose homeostasis, cognitive, renal and hepatic function, haemorrhagic stroke and cataract. Eur Heart J. 2018;39(27):2526-2539.

[49] Chowdhury R, Khan H, Heydon E, et al. Adherence to cardiovascular therapy: a meta-analysis of prevalence and clinical consequences. Eur Heart J. 2013;34 (38):2940-2948.

[50] Descamps OS, Marc C, Patrizio L, et al. Réaction des sociétés scientifiques et des associations de patients face à une émission d'ARTE "le cholestérol, le grand bluff !". Louvain Med. 2018;137(3):397-404.

[51] Stroes ES, Thompson PD, Corsini A, et al. Statinassociated muscle symptoms impact on statin therapy European atherosclerosis society consensus panel statement on assessment, aetiology and management. Eur Heart J. 2015;36:1012-1022.

[52] Cesena FHY, Laurinavicius AG, Valente VA, et al. Statin eligibility in primary prevention: From a risk-based strategy to a personalized approach based on the predicted benefit. Am J Cardiol. 2018;121 (11):1315-1320. 